\title{
Large Field Photogrammetry Techniques in Aircraft and Spacecraft Impact Testing
}

\author{
Justin D. Littell Ph.D. \\ ATK Space Systems \\ NASA Langley Research Center MS 495 \\ 12 W. Bush Rd. \\ Hampton, Virginia 23681 \\ Justin.D.Littell@nasa.gov
}

\begin{abstract}
The Landing and Impact Research Facility (LandIR) at NASA Langley Research Center is a $240 \mathrm{ft}$. high A-frame structure which is used for full-scale crash testing of aircraft and rotorcraft vehicles. Because the LandIR provides a unique capability to introduce impact velocities in the forward and vertical directions, it is also serving as the facility for landing tests on full-scale and sub-scale Orion spacecraft mass simulators. Recently, a threedimensional photogrammetry system was acquired to assist with the gathering of vehicle flight data before, throughout and after the impact. This data provides the basis for the post-test analysis and data reduction.

Experimental setups for pendulum swing tests on vehicles having both forward and vertical velocities can extend to $50 \times 50 \times 50$ foot cubes, while weather, vehicle geometry, and other constraints make each experimental setup unique to each test. This paper will discuss the specific calibration techniques for large fields of views, camera and lens selection, data processing, as well as best practice techniques learned from using the large field of view photogrammetry on a multitude of crash and landing test scenarios unique to the LandIR.
\end{abstract}

\section{Background}

The Landing and Impact Research Facility (LandIR) at NASA Langley Research Center is a $240 \mathrm{ft}$. high by $400 \mathrm{ft}$. long gantry structure. The facility was originally built in 1965 to train the Apollo astronauts for lunar landings [1]. Following the Apollo program, the Lunar Landing Research Facility (LLRF) was converted to the Impact Dynamics Research Facility (IDRF) for use as a full-scale aircraft crash test facility [2]. To date, over 150 vehicles comprising a mix of general aviation aircraft, helicopters, and fuselage subsections have been tested at the recently renamed LandIR. [3] Currently, the facility supports NASA's Constellation and Subsonic Rotary Wing programs for Orion crew module landing and rotorcraft impact testing.

Due to the size of the vehicles tested at the LandIR (commonly known as and referred to herein as the Gantry), all testing is conducted outdoors, either under the $240 \mathrm{ft}$ high A-frame structure or a $70 \mathrm{ft}$ vertical drop tower structure depending on the type of test. Figure 1 shows pictures of the LandIR facility and of the $70 \mathrm{ft}$ drop tower. 


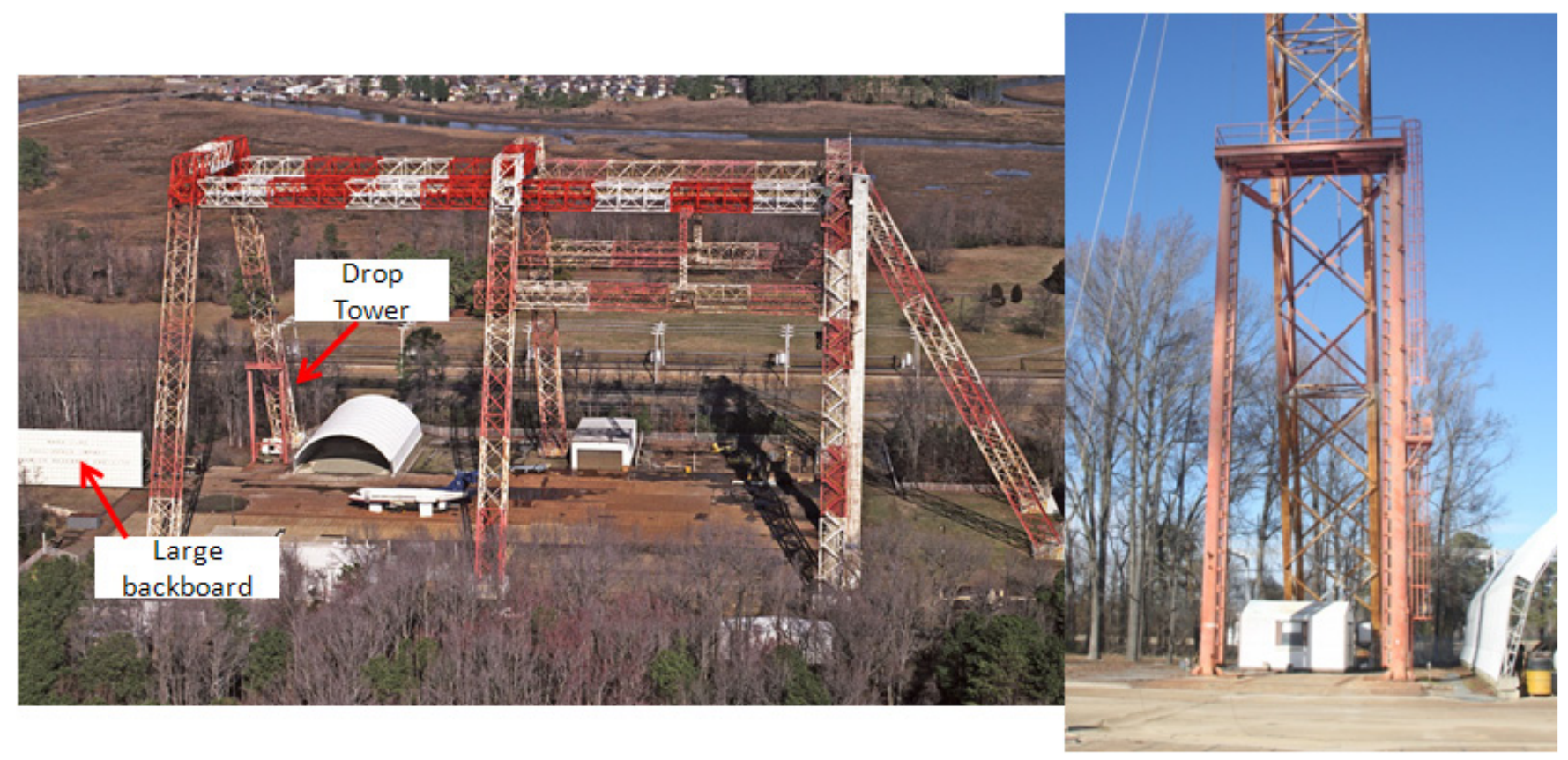

Figure 1 - Landing and Dynamics Research Facility (LandIR) (left), and 70' vertical drop tower (right)

The large size and scale of tests conducted requires particular care for the experimental photogrammetry setup because aspects typically not seen in a controlled laboratory environment must be considered. For further reading on test setups at the Gantry, a detailed description on test preparation and experimental methods can be found in ref. [2]. Ever changing factors in the weather such as wind, clouds, and time of day can affect lighting conditions, along with camera exposure values and aperture settings. Since no two tests are alike, once the test article is moved to the test site, final test preparations can take anywhere from two to four hours. The preparations typically involve various vehicle cabling and roping safety attachments, pyrotechnics placement and arming procedures, final data acquisition system checkouts, and leveling and balancing the vehicles before the final lift, all of which could be happening simultaneously. These items are all mentioned because they can involve personnel and/or equipment such as forklifts or cranes interfering with the photogrammetry field of view from minutes to hours, interfering with camera setup time and/or potentially moving cameras from their calibrated position. Finally, as all tests conducted use very large field of views, typical methods of obtaining conventional photogrammetry calibrations with hand held panels do not work, so alternative calibration procedures are needed. This paper will describe the methods used at the LandIR facility to overcome the above mentioned constraints to obtain well conditioned calibrations with specific examples and results. This paper will focus on applications of a photogrammetry system, rather than a discussion on the theoretical basis behind the specific algorithms used in photogrammetry.

Photogrammetry, in generalized terms, is the analysis to determine geometric properties and specific spatial information of photographed objects. It has been historically used for terrain mapping, civil engineering, accident investigations and automotive crash testing. The automotive industry has been using various forms of photogrammetry in automotive crash tests for the determination of occupant motion [4] and vehicle deformation [5] for many years. Large field photogrammetry has been used for terrain mapping for Geographic Information Systems (GIS), and numerous articles are available in the literature describing features and functions [6][7][8]. In aerospace applications, two dimensional photogrammetry has been used in the past for impact testing of Crew Exploration Vehicle capsules [9], while three-dimensional photogrammetry has been used on solar sails and gossamer structures for dynamic characterization [10]. The objective of this paper is to familiarize the reader with the typical setup steps, constraints, challenges, and lessons learned from outdoor large field three-dimensional photogrammetry, while providing specific examples and test setups, where applicable.

\section{LandIR Calibration Procedures}

The LandIR facility has two types of custom made large field calibration objects. Purely vertical impact tests conducted under the $70 \mathrm{ft}$ drop tower mainly use a portable calibration backboard, whereas full-scale swing tests 
conducted under the Gantry use a calibration grid present on a large backboard. The portable backboard is on rollers, making it easy to move and position to cater to each specific test. The large backboard is only partially movable along railroad tracks in the Gantry's east/west (shown left to right in Figure 1) direction only. Each calibration object has an array of targets positioned at known distances apart. Each target has arc segments appearing at specified angular locations around a center dot such that no two targets have the same "code". The large backboard has 24 coded targets, placed in a $50 \mathrm{ft}$. $\times 36 \mathrm{ft}$. array, while the small portable backboard has 16 coded targets, arranged in a $10 \mathrm{ft}$. $x 6 \mathrm{ft}$. array. Figure 2 shows both calibration objects.

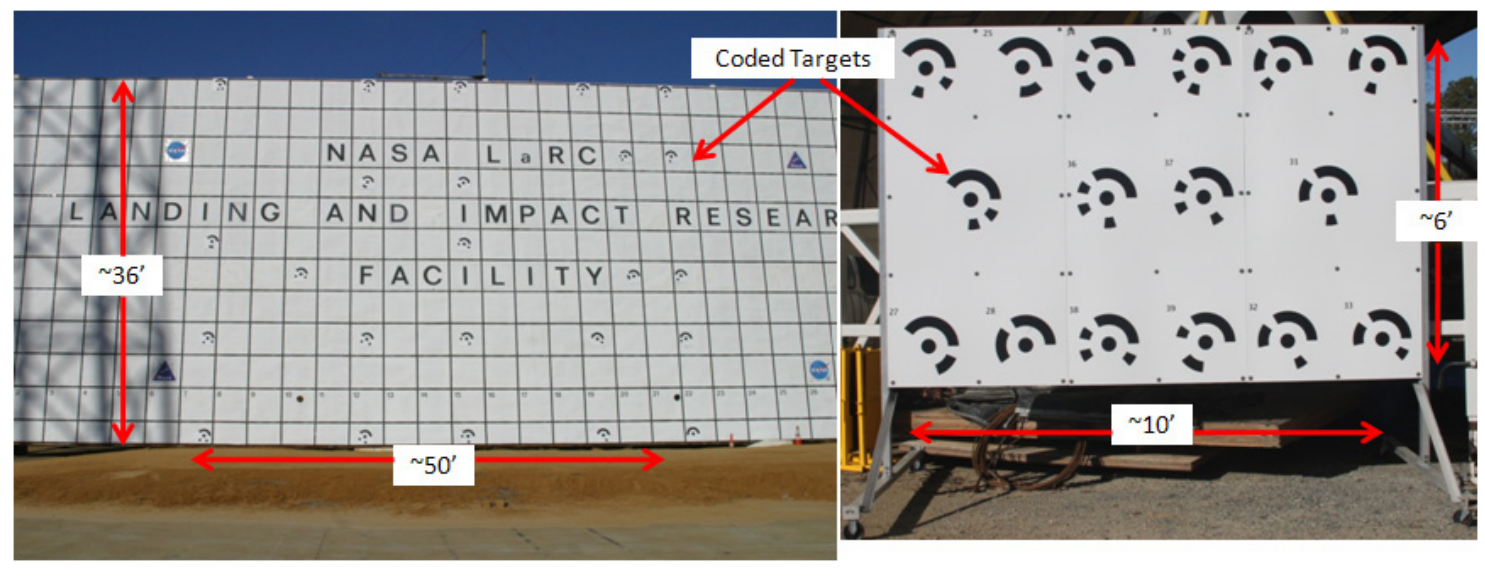

Figure 2 - Large (left) and portable (right) calibration backboards

As with the smaller field calibration procedures, the large field calibration method starts with first determining the approximate size of the field of view needed and determining the lens sizes required. Once the field of view has been determined and the lens has been selected, the technique used for the portable and large backboards is largely the same. Three-dimensional photogrammetry, unlike two-dimensional photogrammetry, uses two or more cameras to create a three-dimensional calibrated volume unique for each test. The off-axis viewing angle of the volume by each camera allows the third, or out of plane, dimension to be triangulated. All three-dimensional photogrammetry techniques require three basic steps for proper calibration, which will be briefly mentioned here. A full procedure can be found in ref [11]. The first step is the removal of camera lens distortion. Camera lens distortions become evident when a circular lens is placed on a camera using a rectangular sensor, and is inherent to varying degrees in all cameras. Parts of the image near the edge of the frame will have a slight curvature about them, in which straight lines will no longer be completely straight. These nonlinear and generally radial lens distortions can be controlled using optical algorithm corrections. Secondly, a three-dimensional "cube", or volume in space, must be generated. Ideally, this region will be comprised of a defined space around the test article's impact location, with enough distance next to or behind the impact location for the placement of static points to establish a reference frame or coordinate system. Finally, the procedure must establish fixed locations for each camera when viewing the newly created calibrated volume. The location and focus for each camera cannot change once these locations have been established. Steps two and three are used in an algorithm typically known as the bundle adjustment. This algorithm uses the pictures and user definitions of known distances in the pictures to determine three-dimensional coordinates of the coded targets in space during the calibration procedures.

All tests were filmed with Phantom 9 cameras at a rate of 1000 frames per second at full camera resolution (1632x1200 pixels). Care was taken to maintain camera settings such as aperture settings, focal length, exposure, etc. the same for the calibration pictures as used for the test. To obtain large field of views under current safety requirements, cameras with $24 \mathrm{~mm}$. lenses were for swing tests. Typical full-scale swing setups required that the cameras be positioned at least $50 \mathrm{ft}$. from the test articles (and $80 \mathrm{ft}$. to $90 \mathrm{ft}$. from the large backboard). Focal depth was set to infinity such that both the test article and backboard were in focus. This configuration typically gave field of views for the full-scale swing tests of approximately $62 \mathrm{ft}$. wide by $45 \mathrm{ft}$. tall and greater than $30 \mathrm{ft}$. for the depth. Tests are normally conducted with the backboards in the cameras field of views such that some of the coded targets used for calibration can also be used for coordinate/reference frame definitions. 
All cameras (along with all other data acquisition equipment used for each test) were connected to an IRIG-B time code generator. The IRIG-B allows data collected on multiple, otherwise incompatible, systems to be synchronized to a common time stamp to facilitate post-test data reduction and post processing. Thus, results presented in the following sections will be plotted against the seconds unit of IRIG time.

When calibrating, a set of 16-25 pictures was taken with each camera. Due to the large size of the calibration objects and unlike smaller calibration procedures, the large field calibration procedure fixed the calibration backboard and moved the camera instead. A series of four to eight pictures was taken with the target array centered and at the extreme edges of the camera's field of view to compensate for the camera lens distortion. The next set of ten to twenty pictures was taken to obtain the calibrated volume. This series acquired images of the backboard from each camera from as much as 40 degrees off axis to the left and $30 \mathrm{ft}$. away from the backboard to 40 degrees off axis to the right and $120 \mathrm{ft}$. away from the backboard, with images being captured at regular intermediate intervals in a grid-like pattern. Finally, each camera was mounted in a fixed location, where it remained for the entire test.

Outside lighting and contrast was determined to be extremely important for each calibration. In varying lighting conditions, the photogrammetry software handled images with flat contrast much better than images with sharp contrast, as illustrated in Figure 3. Normally, sharply contrasting images are desired when finding and tracking targets. Shadowing from the gantry structure, clouds, and supports cables pose unwanted dark spots that are not easy mitigate. Camera settings were established prior to impact testing for bright sunshine or cloudy conditions. However, the conditions can change from sunny to cloudy or vice verse from the time the cameras were set until the impact occurred. Setting up for cloudy conditions is preferred for photogrammetric measurements because shadowing is minimized. Sharp contrast imaging is most desired for qualitative observations and visual inspections.
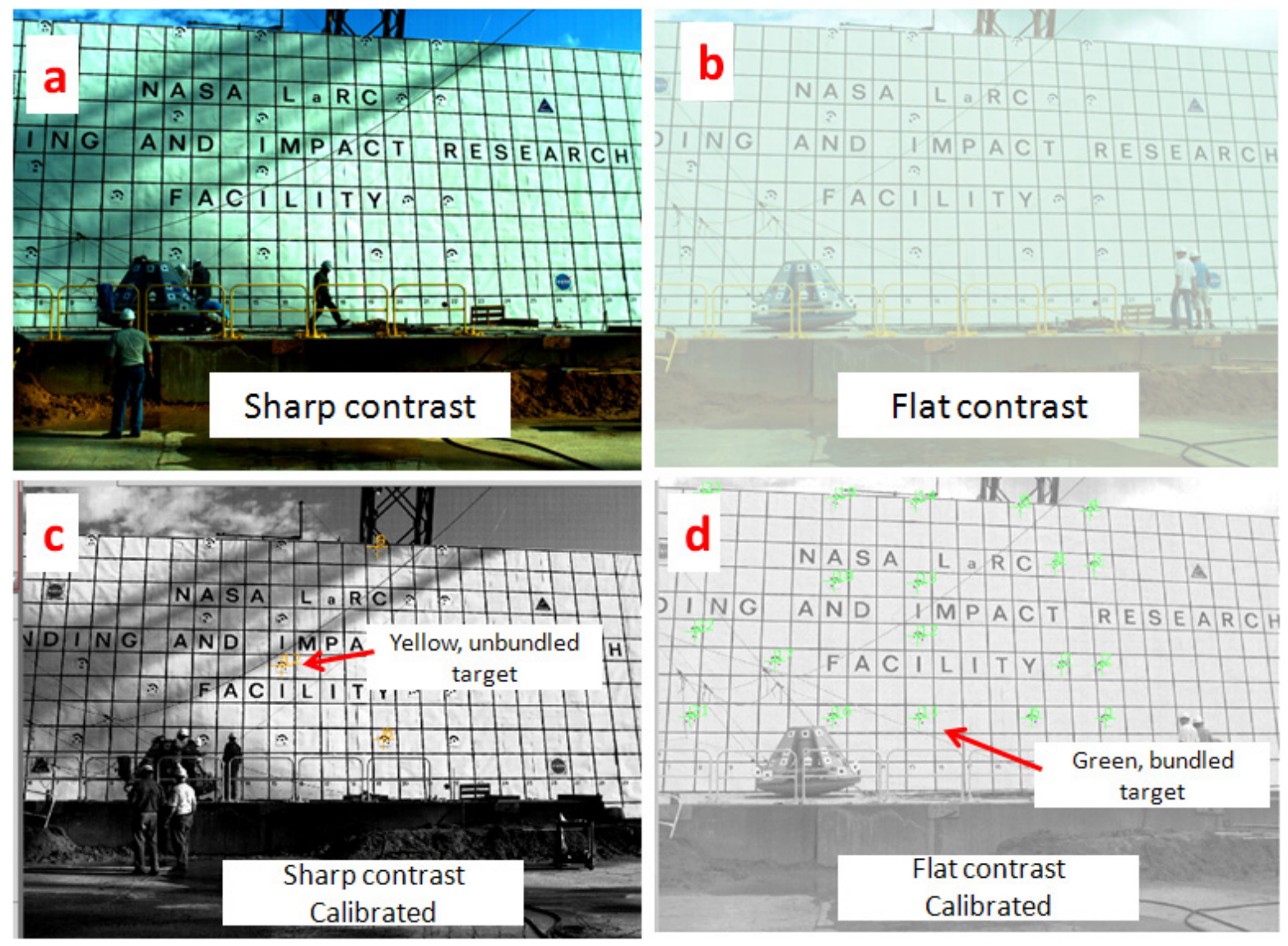

Figure 3 - Calibration findings using sharp contrast (left two images) and flat contrast (right two images)

Figures 3(a) and 3(b) are from the final mounted location of the left camera taken approximately a half hour apart during the morning of a sunny day in the late summer of 2009. The sun position and lighting conditions were 
approximately the same for both figures. Figure 3(a) shows a shadow from one of the Gantry legs on the large backboard taken at the camera contrast used for a typical test. The shadow is obscuring approximately one third of the target array, mainly in the upper left corner. The image in Figure 3(b) is taken with the contrast artificially adjusted, whereas all of the other test variables were the same. Figures 3(c) and 3(d) are screenshots from the photogrammetry calibration software. Figure 3(c) shows the identification of three of the coded targets on the array displayed in yellow. Yellow indicates that the three targets were identified but the bundle adjustment failed to converge the coordinates of the targets between the left and right (view not shown) cameras. However, the calibration shown in Figure 3(d) identifies the majority of targets. In addition the green color indicates that the bundle adjustment has occurred successfully. It is believed that by adjusting the contrast on the images to give an artificially flat result allows for the software pattern recognition capabilities to resolve areas between light and dark more easily if all of the lighter areas are of the same lightness and all of the darker areas are of the same darkness. For the sharply contrasted images, Figures 3(a) and 3(c), the contrast between the light and dark areas for those targets in the shadow is different than the contrast in light and dark areas for those targets in the sun. This difference leads to misidentification and/or no identification from the software. After this finding, care has been taken to artificially flatten all calibration (and test) images on bright and sunny days. During cloudy days, the clouds artificially flatten the images, so no adjustments are made.

\section{Experimental Setups}

Since 2009, three-dimensional photogrammetry has been used on a series of full-scale impact tests conducted at the LandIR facility for both NASA's Constellation [12] and Subsonic Rotary Wing [13] Programs. A summary of the specific test setups and preparations used are provided in this section. This section also provides a subset of tests to illustrate the capabilities and limitations of a photogrammetry system seen on large scale testing conducted on aircraft and spacecraft. The section will start with the simplest setups and proceed to the most involved, and do not necessarily follow chronological order.

\section{MD-500 Mass Simulator Swing Testing}

One of the first instances where three-dimensional target tracking photogrammetry was used was on an impact test of a helicopter mass simulator. The test article consisted of a $3,000 \mathrm{lb}$ aluminum flat plate with skid gears, representing an approximate test configuration of a 3,000 lb MD-500 helicopter. This mass simulator was used to obtain baseline accelerations when tested with a foam block under the plate acting as an impact energy attenuation mechanism. In addition, the test article contained a replacement shock absorbing strut as the interface between the skid gear and airframe. The plate was instrumented with 32 channels recording accelerations at various locations and 11 photogrammetric targets. Figure 4 shows the experimental setup with camera locations, test article and calibrated volume highlighted. 


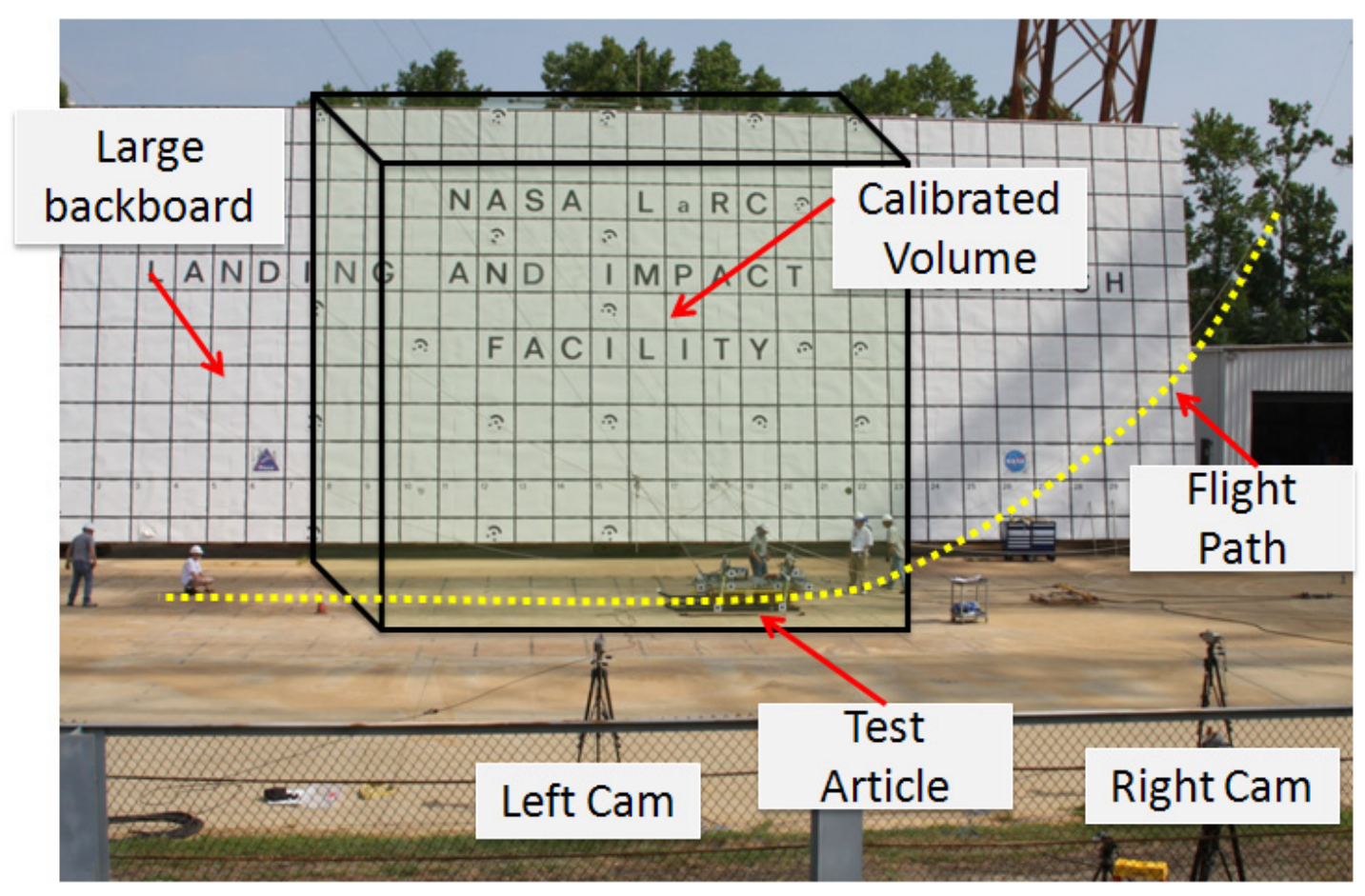

Figure 4 - MD-500 Mass simulator full-scale swing test setup

The flight path of the vehicle was from the right to the left as shown by the yellow line in Figure 4 . The test article is positioned at the approximate impact location in the figure. Most of the calibrated volume was to the left of the impact location, which allowed for post-impact slide out data to be collected. Figure 4 also shows the cameras in their fixed position for the test, 50 feet away from the test article, 80 feet away from the large backboard and approximately 24 feet apart. These dimensions result in a 15 degree angle to the normal direction of the vehicle's flight path.

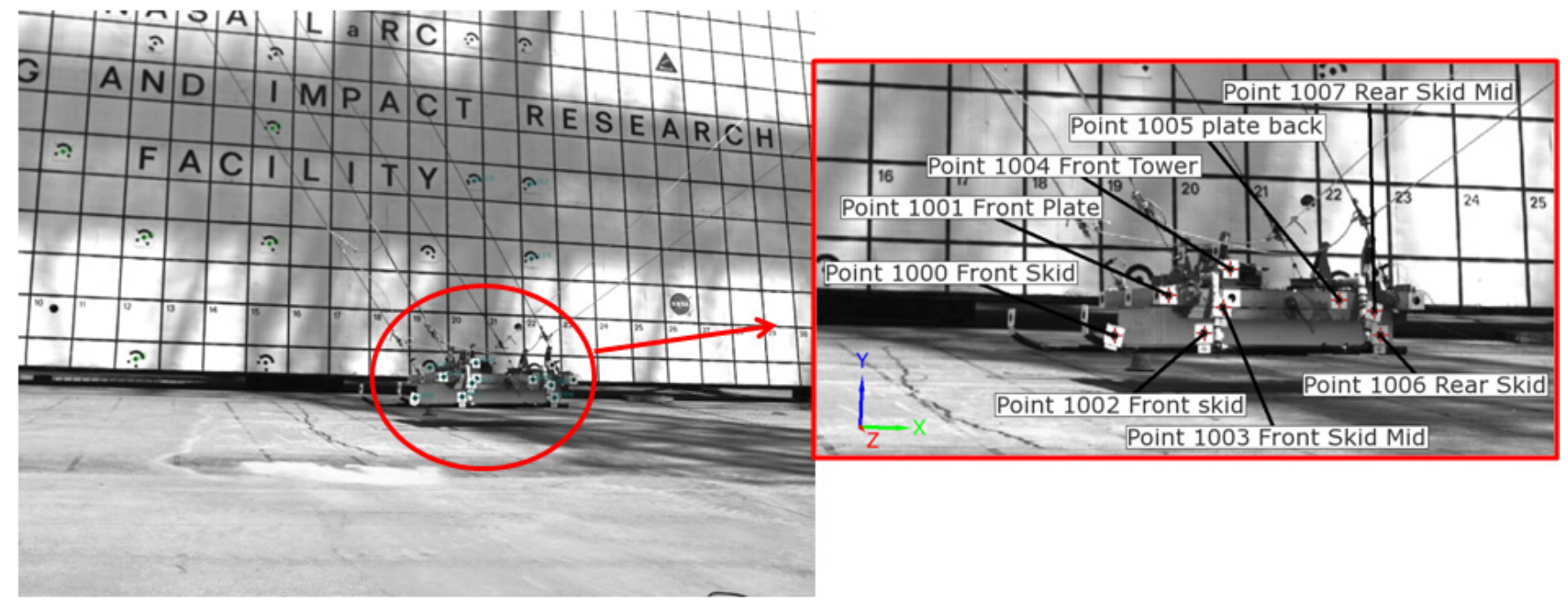

Figure 5 - Flat plate swing test full view (left) and close up showing target locations/identifications (right)

Figure 5 shows a frame of the high speed video from the left camera immediately before test article impact and subsequent target identification as processed by the photogrammetry system. One target in Figure 5 was not tracked, which was a consistent problem in subsequent frames of data for this specific test. This target was located below Point 1004. The large relative motion between the skid gear and the flat plate caused some of the targets on the skid gear (Points 1000, 1002, 1003, 1006, 1007) to overlap some of the targets on the plate (Points 1001,1004 , and 1005). A major lesson learned from this test was that targets should not be placed too close 
together and thus interfere with identification during impact. An additional item of interest learned specifically from this test was that the targets on the skid gear were attached to the gear by a series of flexible standoffs, which caused the targets to oscillate. Figure 6 shows post impact lateral displacement comparison between the skid gear and flat plate for the first 0.5 seconds after impact. There are very slight oscillations from the flat plate due to the foam compression, but the pronounced oscillations occurred in the target (Point 1000) attached to the front skid gear. The vertical red line represents the time of the vehicle impact. Subsequent tests for this test article and others had the targets rigidly attached to the skid gears or other rigid locations and further apart to avoid overlap and cover.

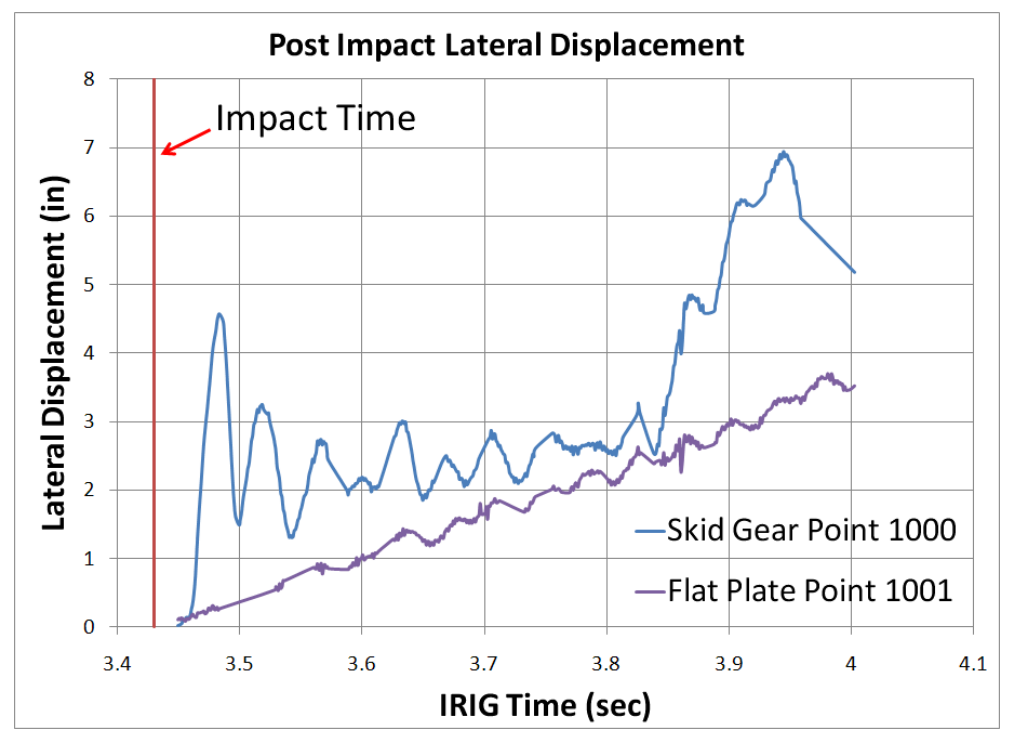

Figure 6 - Relative lateral motion comparison between the skid gear and rigid plate

In order to process the vehicle data, a coordinate system had to be established such that the three orthogonal axes represented the three directions of motion from the vehicle. The coordinate system was created on the large backboard by using the static coded targets present. A coordinate system was created by first defining a plane using three targets, typically those in the corners of the field of view. Then, a positive $\mathrm{x}$ axis was defined using two of the targets lying in the same horizontal plane. Finally, an origin was defined using one of the targets in the lower left hand corner of the backboard. In the coordinate system, the $x$ direction represented horizontal velocity, $y$ direction represented vertical velocity and $z$ direction represented out-of-plane, or lateral velocity. Impact velocities were taken as the average of all the vehicle target points at the time of ground contact as determined by visual inspection of the high speed video. Pitch, roll, and yaw angles were found by creating projection angles in the $x y, x z$, and $y z$ directions using artificially created lines on the vehicle and artificial lines created on the backboard. Figure 7 shows a schematic on the projection angle methodology. Vehicle pitch was determined by calculating an xy projection angle between a horizontal line on the vehicle (Points 1001 to 1005) and a horizontal line on the backboard. Vehicle yaw was calculated by creating an xz projection angle between a horizontal line on the vehicle and a horizontal line on the backboard. Vehicle roll was calculated by creating a yz projection angle between a vertical line on the vehicle and vertical line on the backboard. All of these angles can be differentiated (by the software) to give angular rates at impact as necessary. 


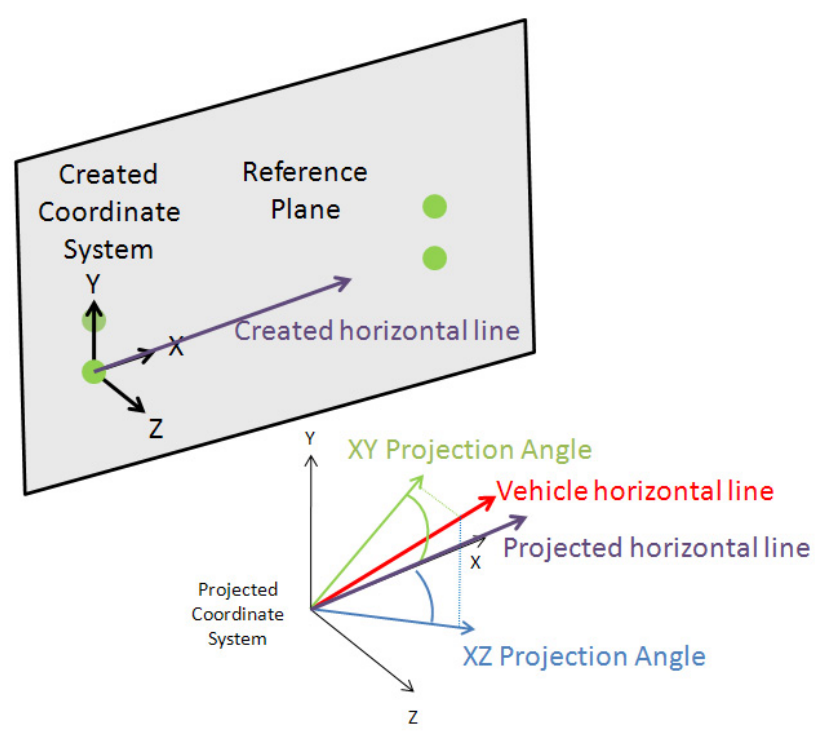

Figure 7 - Projection angle methodology

For the MD-500 mass simulator, the impact velocities were computed to be $27.1 \mathrm{ft} / \mathrm{sec}$ horizontal and $17.5 \mathrm{ft} / \mathrm{sec}$ vertical with 1.8 degree pitch, 6.7 degree yaw and 3.4 deg roll angles. These values deviate from the planned conditions of $28 \mathrm{ft} / \mathrm{sec}$ horizontal, $18.2 \mathrm{ft} / \mathrm{sec}$ vertical velocities with 0.0 degree pitch, roll and yaw angles.

Finally, target displacements on the large backboard were checked. It was assumed that the large backboard and targets attached to large backboard remained stationary during tests. As such, the amount of displacement computed by the photogrammetry software was the noise level, or the amount of uncertainty in the measurements. Three targets were picked to examine the noise levels; $11,13,16$, because they represented a large area of the backboard far enough away from the mass simulator test article and cabling as to remain visible. The target locations and resultant root-sum-squared displacements are shown in Figure 8 . The majority of the data resides within the \pm 0.05 inch range for all three targets. This 0.10 inch noise range over the length of 62 feet represented a $0.01 \%$ error in the data.
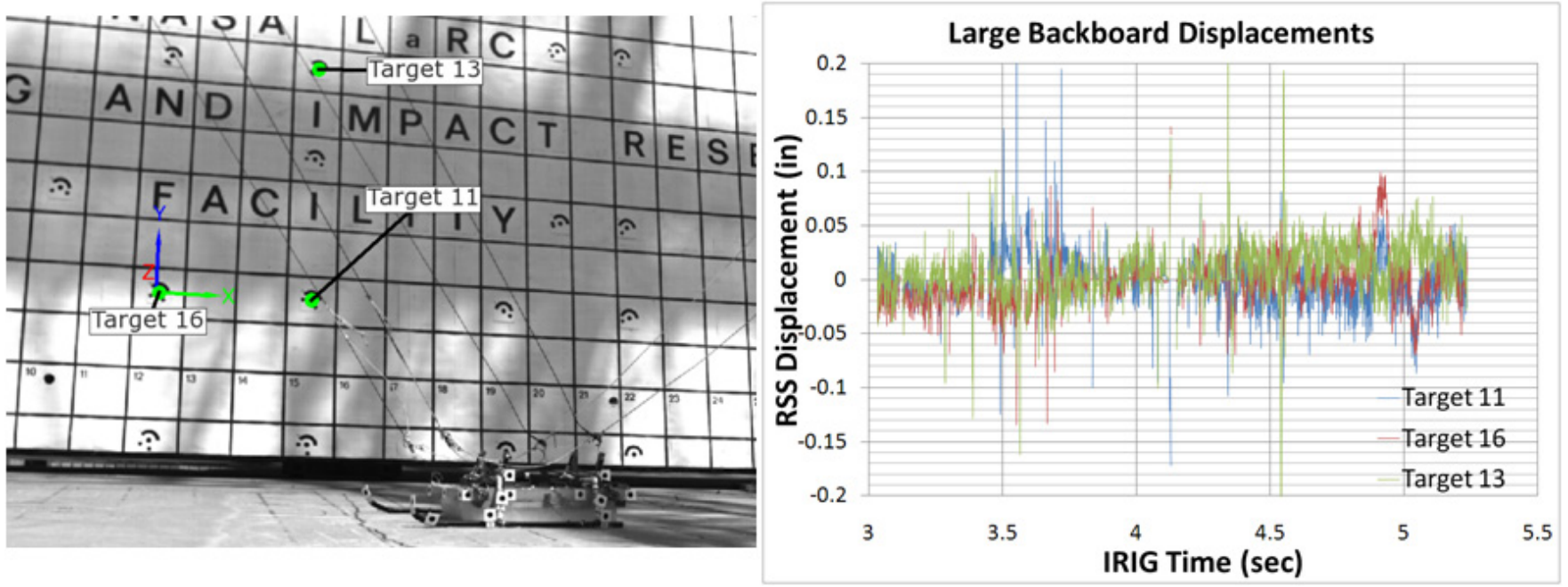

Figure 8 - Backboard Target Locations (left) and displacements (right) 


\section{Subscale Orion Swing Testing}

A series of 5 swing tests was conducted by the Constellation program in the summer of 2009. This program tested a $5146 \mathrm{lb}, 8 \mathrm{ft}$. diameter Subscale Orion mass simulator boilerplate to assess abort land landing conditions. The Subscale Orion impacted a $4 \mathrm{ft}$. high by $20 \mathrm{ft}$. wide by $78 \mathrm{ft}$. long sand bed at various vertical and horizontal velocities to assess the effects of horizontal velocity in the vehicle response. Capsule deformations were not considered for these tests as the capsule was assumed to be a rigid structure. This series of tests, however, brought about increasing photogrammetric complexity because the fabricated sand bed obscured the bottom row of coded targets on the large backboard. After several attempts to calibrate, the problem was remedied by taking extra calibration pictures such that the first visible row of calibration targets on the backboard was captured near the bottom of the cameras' sensors to ensure that lens distortions were removed. Then, the cameras were tilted slightly such that the bottom of the calibrated cube would be on the surface of the sand bed approximately $4 \mathrm{ft}$. off the ground. Figure 9 shows a picture of the test setup taken from the right photogrammetry high speed camera. Note that the image has been artificially flattened as described previously to give an even white/black contrast on the entire backboard.

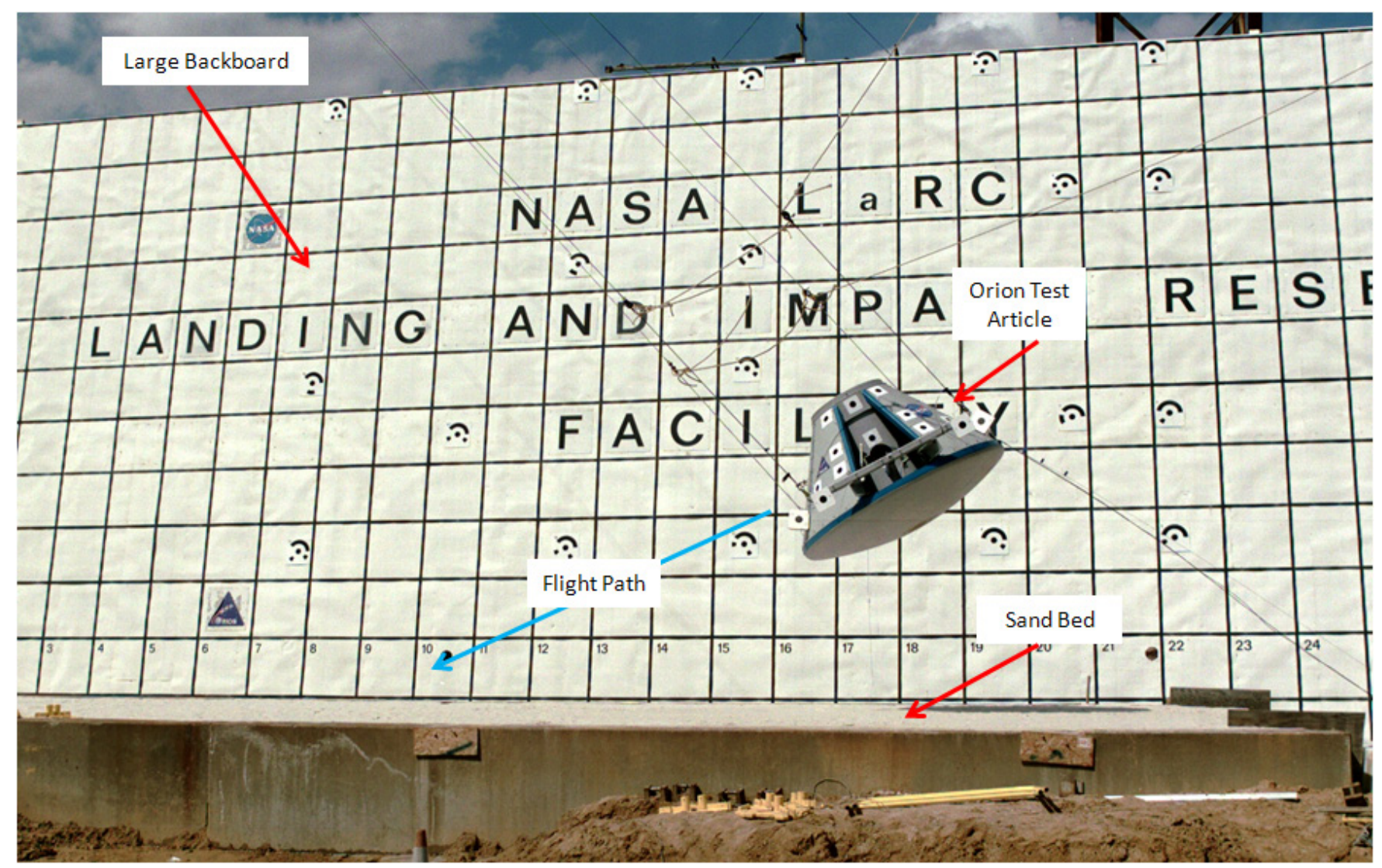

Figure 9 - Test setup for the Subscale Orion Swing tests

Ten targets were located on the vehicle. Eight of these targets were located on the vehicle's skin, and two were located on an out-rigging bar, which provided the Gantry's lifting/swinging interface point. Often times when conducting full-scale swing tests, extra lifting and/or swinging hardware is added to facilitate interface with gantry cabling systems. This extra hardware is ideal for photogrammetry target placement as these points can be thought of as rigid locations which minimally deform. Figure 10, left, shows the tracked targets on the Subscale Orion while Figure 10, right, shows approximately $1 / 2$ second (500 images) of vehicle velocity time history data. For the Subscale Orion test presented, the vehicle impacted the soil at $29.8 \mathrm{ft} / \mathrm{sec}$ horizontal, $24.7 \mathrm{ft} / \mathrm{sec}$ vertical and $0 \mathrm{ft} / \mathrm{sec}$ lateral velocities, corresponding with the nominal $30 \mathrm{ft} / \mathrm{sec}$ horizontal, $25 \mathrm{ft} / \mathrm{sec}$ vertical and $0 \mathrm{ft} / \mathrm{sec}$ lateral velocities. 

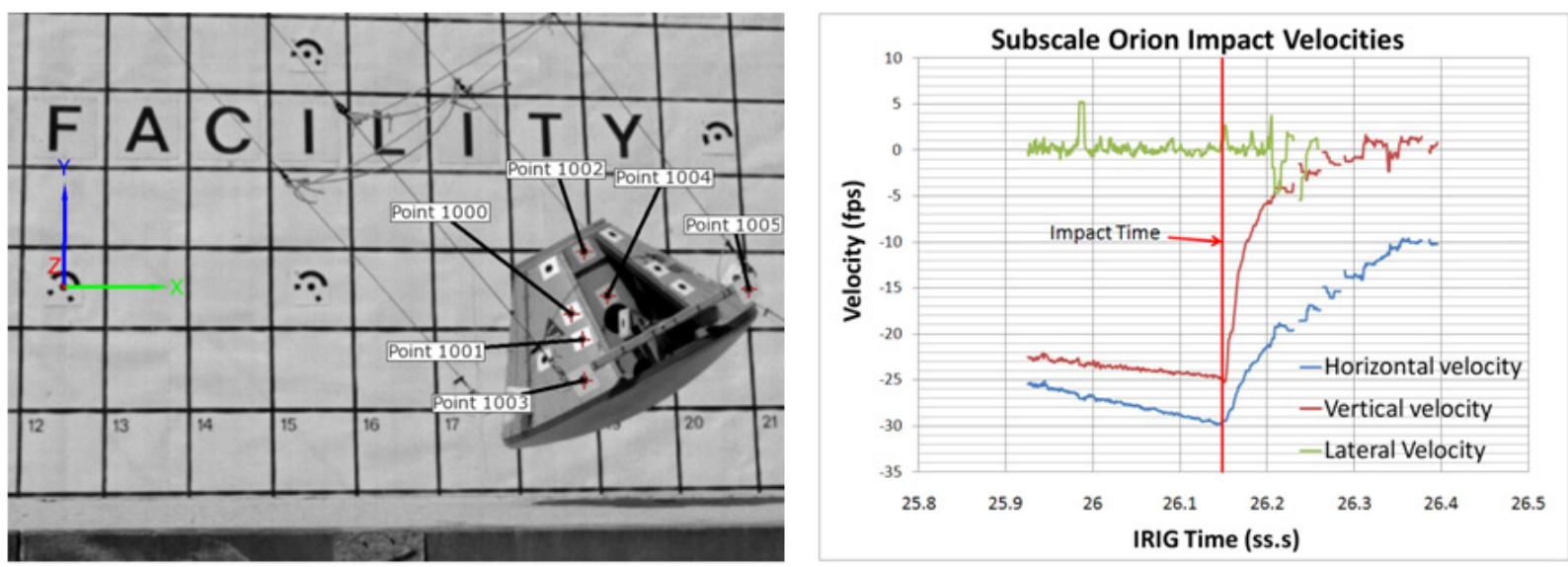

Figure 10 - Tracked targets on the Subscale Orion (left) and impact velocities (right)

\section{MD-500 Full-Scale Swing Test}

Photogrammetry techniques were used for a full-scale crash test of an MD-500 helicopter in December 2009. This crash test was the most complex in that both the target tracking and full field strain capabilities of the system were implemented. The purpose of this test was to evaluate a prototype composite energy absorbing concept to reduce the risk of crew injury during accidents. As a result, vehicle deformations and impact conditions were critical to evaluate the energy absorber's capabilities. The helicopter was instrumented with 160 data channels recording strain, acceleration, load and occupant data, but also instrumented with a grid of targets on the side of the airframe, along with targets on the tail, rotor mount, skid gear and belly to record both vehicle impact conditions and also gross vehicle deformation. As a proof of concept, the tail skin was also painted with a white/black speckle pattern to enable full-field strain photogrammetry. Targets used for the target tracking photogrammetry were approximately 3 in. diameter, whereas the black dots used in the full-field strain photogrammetry were $1 \mathrm{in}$. diameter. Figure 11 shows a picture of the instrumented helicopter.

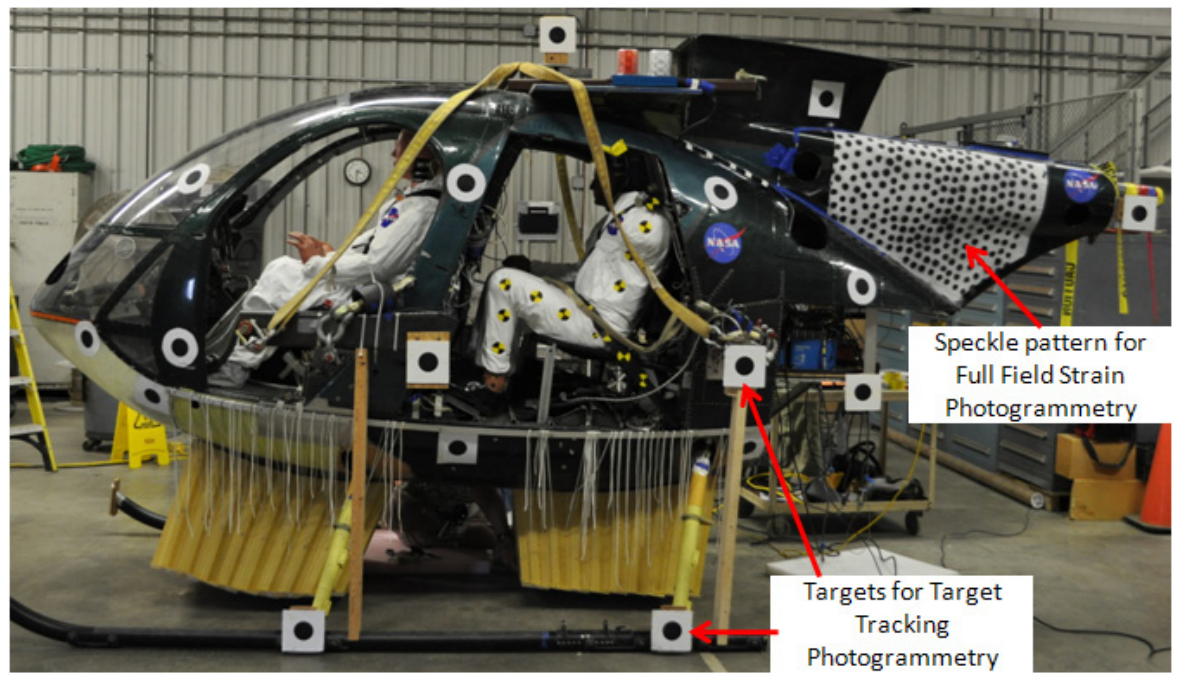

Figure 11 - Fully Instrumented MD-500 helicopter

Information to assess the helicopter's impact velocities along with pitch, roll and yaw impact angles was ultimately needed. However, because the helicopter was considered a deformable structure, structural deformations also were of interest to assess the integrity of the airframe post-impact. Figure 12 shows the un-coded targets tracked on the vehicle (Points 1000 - 1013), static coded targets found on the backboard (Point $6,8,12,13,15,16,17$, 18,21 , and 22) and the coordinate system created (lower left hand corner of Figure 12, right) 

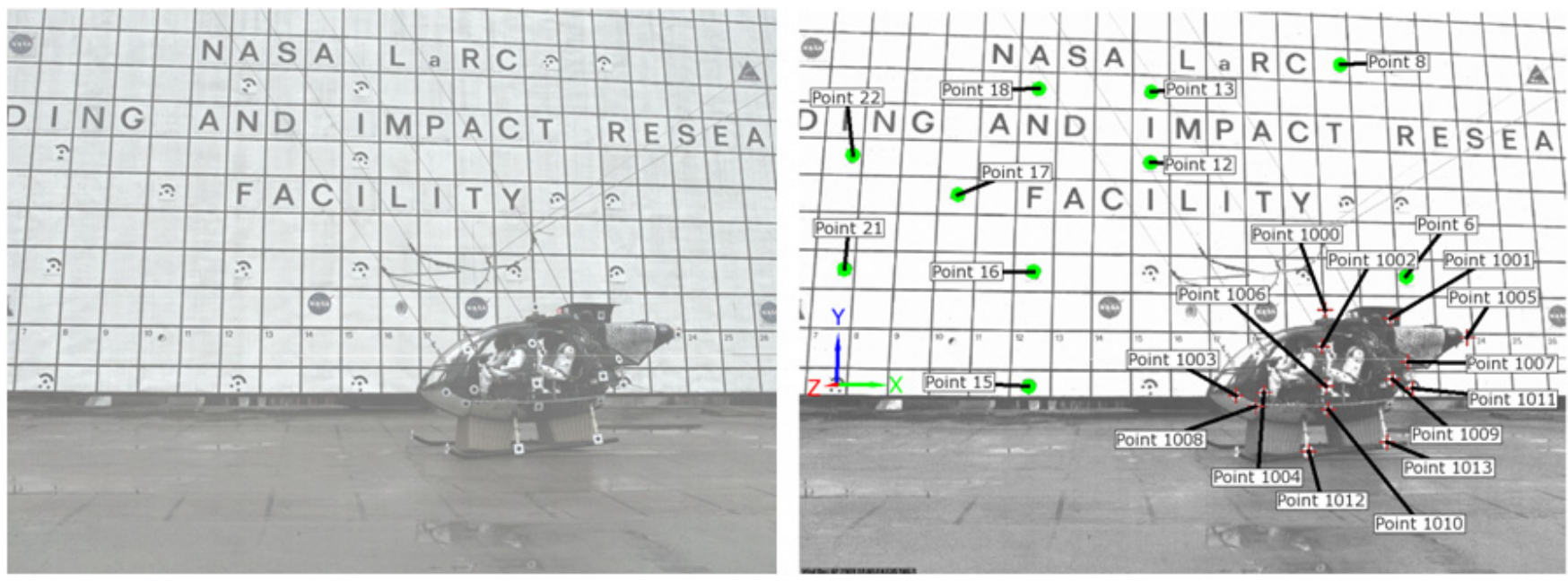

Figure 12 -Single image captured from high speed movie (left) and post processed photogrammetry data (right)

As with the Subscale Orion tests, impact velocity conditions were determined from an average of all points on the vehicle at impact, while pitch and yaw angles and angular rates were taken from an average of $x y$ and $x z$ projected angles from the lifting hardware (Points 1006 and 1009) and skid gear (Points 1012 and 1013) with respect the horizontal axis, defined by the $x$ axis in Figure 12. Roll angle and angular rate were obtained from measuring the angle of a yz projected line. This measurement was more complicated, since no two targets were in the same vertical plane on the vehicle. By using Points 1006 and 1002 and subtracting out the built- in angle on the vehicle, roll parameters were computed. The photogrammetric results showed the vehicle impacted at $38.7 \mathrm{ft} / \mathrm{sec}$ horizontal and $25.5 \mathrm{ft} / \mathrm{sec}$ vertical velocities, with 5.7 degrees pitch, 9.3 degrees yaw and 7.0 degrees roll with angular rates of $4.8 \mathrm{deg} / \mathrm{sec}$ yaw and $1.1 \mathrm{deg} / \mathrm{sec}$ roll. Figure 13 shows these results, along with results for about $800 \mathrm{~ms}$ after vehicle impact. The red vertical line is included to show the impact time (4.605 seconds).
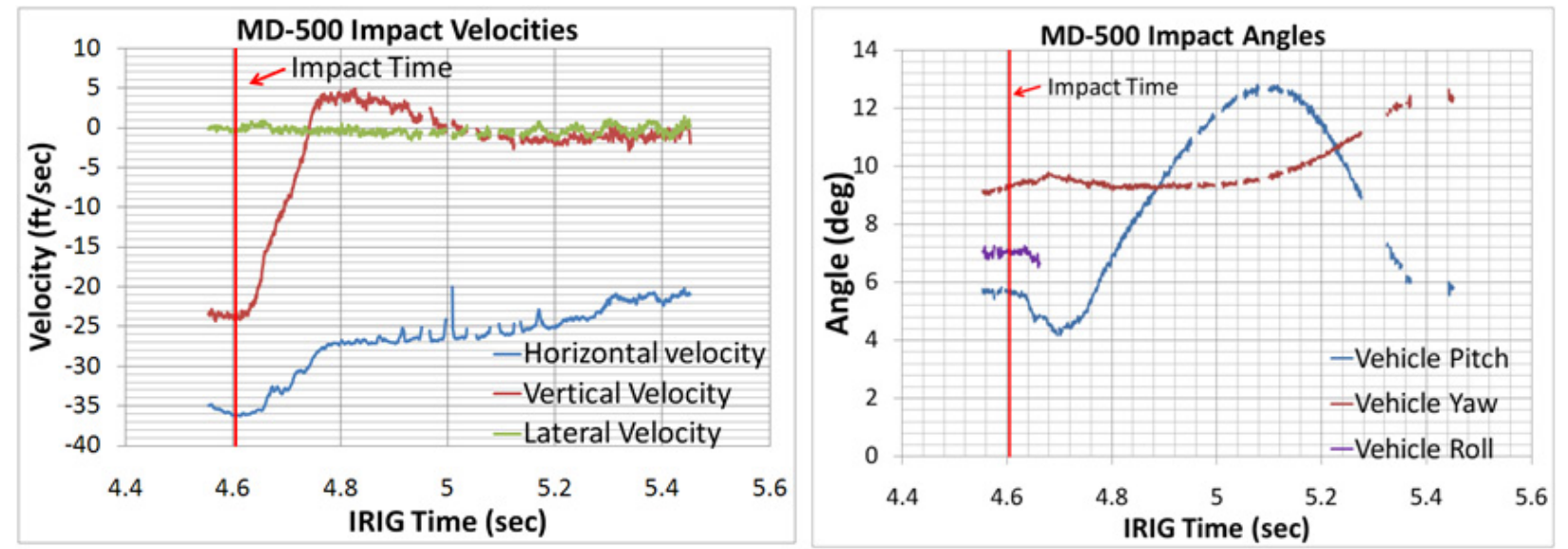

Figure 13 - Impact conditions of the MD-500 helicopter

Care was taken to locate specific target locations on the vehicle such that the structural integrity of the vehicle could be analyzed post test. The targeting scheme can be summarized as follows: two targets measured the relative motion of the skid gear (Points 1012 and 1013), three targets measured the waterline deformation of the vehicle (Points 1003, 1008, 1010), four points formed a horizontal line in-line with the lifting hardware (Points $1003,1004,1006,1009)$ and created the "seat" line for the occupants, three points defined the helicopter's nose deformation (Points 1003, 1008, 1004), two points define the structural integrity of the data acquisition system mounting hardware (Points 1007, and 1011), and separate points define the locations for the top rotor mass ballast (Point 1000), occupant "head" line (1002), rear engine fairing (Point 1001) and rear tail (Point 1005). Note that some of the targets were used for multiple functions. Structural integrity of the airframe was determined by examining the time history of the change in distance between the various targets on the airframe. The maximum deformation was found (and reported as strain) while the time history was examined to determine if the maximum 
deformation stayed constant at the max value, or began to unload toward a zero value. Target distances and maximum permanent deformations are listed in Table 1.

Table 1 - Deformation Measurements of the MD-500 helicopter

\begin{tabular}{|l|c|c|c|}
\hline Location (Target Numbers) & Nominal Distance (in.) & Max Strain (\%) & Permanent \\
\hline Nose to Water Line $(1003-1008)$ & 14.14 & 0.85 & No \\
\hline "Seat" line, Nose $(1003-1004)$ & 17.80 & 0.47 & No \\
\hline Water Line $(1008-1010)$ & 47.48 & 0.17 & No \\
\hline DAS Shelf (1007-1011) & 17.34 & 0.98 & No \\
\hline $\begin{array}{l}\text { Top mass to front lifting fixture } \\
(1000-1006)\end{array}$ & 64.70 & 0.32 & No \\
\hline $\begin{array}{l}\text { Top mass to rear lifting fixture } \\
(1000-1009)\end{array}$ & 66.73 & 0.40 & No \\
\hline $\begin{array}{l}\text { Rear Engine Fairing to rear lifting } \\
\text { fixture (1001-1009) }\end{array}$ & 54.80 & 0.24 & No \\
\hline $\begin{array}{l}\text { Tail to rear lifting fixture (1005 - } \\
1009)\end{array}$ & 83.79 & & \\
\hline
\end{tabular}

The results in Table 1, along with visual inspections and strain gage data on keel beams and bulkheads, helped to indicate that very little permanent deformation occurred on the airframe, and thus it was suitable for reuse. Areas showing permanent deformation (mainly forward lower skin areas) and buckling have been removed and repaired.

Finally, a proof of concept using the full field strain measurement system was completed on the MD-500 tail skin. This was to evaluate if the dot pattern was acceptable to resolve full field strains on a full-scale vehicle crash test. As a check, a strain gage rosette was wired in the lower quadrant of the tail skin, which was then painted over by the speckle pattern. Results show that the full field strain measurement system was able to resolve a strain field, however the size of the dots were large and did not capture the localized effects captured by the strain gauge rosette. The rosette featured three $0.125 \mathrm{in}$. length gages arranged in a $0 / 45 / 90$ deg arrangement covering an approximate 1 square inch area, whereas the resolved grid pattern of results for the full field strain photogrammetry system was approximately 1.5" apart. Data for each point in the grid pattern used multiple adjacent grid point locations in an averaging function to obtain results for each discrete position, such that the actual gage size for the full field strain measurement was on the order of 6". Future investigations are necessary to address the issues of capturing more localized defects by reducing dot size, decreasing the camera field of view, or using alternate hardware. However, the proof of concept in taking measurements on a large structure was successful.

\section{Discussion and Conclusion}

Large field three-dimensional photogrammetry techniques have been used for approximately 20 full-scale aircraft and spacecraft impact tests for determination of vehicle impact conditions and also structural deformations. Representative individual results for the full six degree of freedom impact conditions have been presented as examples of capabilities of the applied techniques. Specific examples included in this paper are results for Subscale Orion swing tests, impact conditions on MD-500 mass simulator and helicopter swing tests, and structural deformations on the MD-500 helicopter during a full scale crash test.

Since 2009, the LandIR at NASA Langley Research Center has been using three-dimensional photogrammetry for both aircraft crash and spacecraft impact tests. Obstacles faced in conducting photogrammetry setups in outdoor large field of view environments have been described, and solutions to these obstacles have been provided. Mitigation strategies due to specific hardware and facility constraints, such as the immobility of the large backboard and the impact of vehicles above the ground surface were provided. Many of the techniques described were developed in tandem with the testing, basing updated procedures on previous sets of findings and results. For example, backboard target interference due to shadows hindered target recognition, which was resolved by trial and error flattening of the image series. 
Processes to document results in the photogrammetry software for the full six degree of freedom solution were developed in tandem with photogrammetry setup procedures. Target placement techniques have been refined to eliminate overlap and lost data, while a methodology has been developed for optimum placement of targets for angle analyses in two separate planes. Static targets were added to all tests such that coordinate transformation techniques could be implemented. This is especially critical for tests conducted with the large backboard, which was rotated at an angle of 9.4 degrees from the vertical. Finally, noise levels were checked within the measurements by examining backboard fixed target displacements.

\section{References}

[1] O'Bryan, Thomas C., and Hewes, Donald E., "Operational Features of the Langley Lunar Landing Research Facility," NASA Technical Note, TN D-3828, February 1967.

[2] Vaughan, Jr., Victor L. and Alfaro-Bou, Emilio, "Impact Dynamics Research Facility for Full-Scale Aircraft Crash Testing," NASA Technical Note, TN D-8179, April 1976.

[3] Jackson, K.E., et al. A History of Full-Scale Aircraft and Rotorcraft Crash Testing and Simulation at NASA Langley Research Center. NASA TM 20040191337. 2004.

[4] McClenathan, R.V. et al. Use of Photogrammetry in Extracting 3D Structural Deformation/Dummy Occupant Movement Time History During Vehicle Crashes. SAE 2005 World Congress and Exhibition. SAE Paper \# 2005-01-0740. 2005.

[5] Fenton, S. et al. Using Digital Photogrammetry to Determine Vehicle Crush and Equivalent Barrier Speed (EBS). SAE International Congress and Exposition 1999. SAE Paper \# 1999-01-0439. 1999.

[6] Bulner, M.H. et al. How to use Fiducial-Based Photogrammetry to Track large-Scale Outdoor Motion. Experimental Techniques. Pp 40-47. 2010.

[7] Lane, S.N. et al. Application of Digital Photogrammetry to Complex Topography for Geomorphological Research. Photogrammetric Record, 19(95). Pg 793-821. 2000.

[8] Collin, R.L and Chisholm, N.W.T. Geomorphological Photogrammetry. Photogrammetric Record 13(78). Pg 845-854. 1991.

[9] Barrows, D. et al. Photogrammetric measurements of CEV Airbag Attenuation Systems. 46th AIAA Aerospace Sciences Meeting and Exhibit. AIAA Paper 2008-846. 2008.

[10] Black, J.T. et al. Photogrammetry and Videogrammetry methods for Solar Sails and Other Gossamer structures. 45th AIAA/ASME/ASCE/AHS/ASC Structures, Structural Dynamics and Materials Conference. AIAA Paper 2004-1662. 2004.

[11] GOM mbH "PONTOS User Manual - Software" GOM mbH, Germany. 2008.

[12] Baumeister, J. NASA's Constellation Program. NASA/CP-2009-215677. 2009

[13] Jackson, K.E., Fuchs, Y. T., and Kellas, S., "Overview of the NASA Subsonic Rotary Wing Aeronautics Research Program in Rotorcraft Crashworthiness" Journal of Aerospace Engineering, Special Issue on Ballistic Impact and Crashworthiness of Aerospace Structures, Volume 22, No. 3, July 2009, pp. 229-239. 